\title{
Cis-Trans Isomerization of Proline Dipeptides during Liquid Chromatography: Kinetic Analysis of the Elution Profile
}

\author{
Takashi Nishikawa, Yoshinori HayashI, Satomi Suzukı, Hiroaki Kubo \\ and Hideki OHTANI \\ Department of Clinical Pathology, School of Medicine, Kitasato University, \\ Kitasato, Sagamihara, Kanagawa 228, Japan
}

\begin{abstract}
A proline dipeptide, Leu-Pro, was eluted on a reversed-phase liquid chromatographic column with UV detection. Since this peptide undergoes cis-trans isomerization, various chromatographic profiles were produced. With the column kept at a low temperature, two peaks were produced, corresponding to the cis- and the trans-form. With elevation of column temperature or with increase of retention time, the peak area of the non-isomerized molecules was decreasing while the area corresponding to the isomerized molecules was increasing in a complementary manner. With further temperature elevation or with further retention time increase, only a broad peak of the isomerized molecules was produced. We demonstrate that the isomerization velocity could be estimated from the chromatograms and that the peak height of the isomerized molecules was proportional to the square root of retention time. Three other X-Pro dipeptides (Ala-Pro, ValPro, Phe-Pro) showed similar chromatographic behavior that was characteristic for reversible isomerization between two forms.
\end{abstract}

Keywords Proline peptide, peptide bond, cis-trans isomerization, high-performance liquid chromatography

Degradation, isomerization, or any other reactions of the analyte during separation are undesirable for chromatography. However, not a few compounds exist in multiple reversible forms reacting on the time order of minutes, i.e., the time scale of HPLC separation, and their HPLC profiles have been rarely reported. When a reversible reaction such as isomerization occurs, a peculiar chromatogram is obtained and frequently misinterpreted as: (1) impurity in sample produces a minor peak, (2) column deterioration or apparatus misfunction yields peak tailing, spreading, splitting or distortion (3) new isomer appears at a certain temperature, (4) multiple isomers are present at a low temperature while coalescing into one form at higher temperature, (5) dependence of peak sharpness on column temperature or eluent flow-rate is the result of other mechanisms such as eluent viscosity and slow equilibration of compound between mobile- and stationary-phases.

We analyzed HPLC profiles of glucose and some other sugars that undergo $\alpha-\beta$ or pyranose-furanose conversion. ${ }^{1}$ We also demonstrated through the profile analysis that immunosuppressive drugs such as cyclosporins, tacrolimus (FK506) and rapamycin undergo reversible conversion between two forms. ${ }^{2-4}$

Here we analyze HPLC profiles of some proline dipeptides (X-Pro) that constitute another group of convertible compounds. They undergo cis-trans isomerization at their imide bond $(-\mathrm{CO}-\mathrm{N}<)$, while most peptides including prolyl dipeptides (Pro-X) adopt only trans conformation at their amide bond (-CO-NH-) (Fig. 1). ${ }^{5-7}$

Chromatographers are sometimes confronted with peculiar profiles that make them suspicious of on-column conversion. The quantitative analysis of the profile described here will be useful to judge if the peculiar profiles are due to reversible conversion or not.

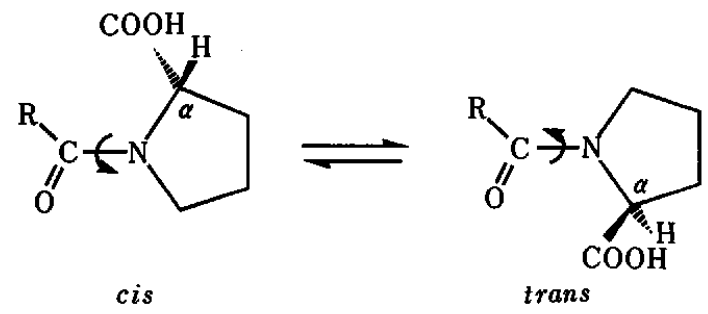

Fig. 1 Cis-trans isomerization of proline dipeptide. 


\section{Experimental}

All the dipeptides, consisting of $\mathrm{L}$-amino acids, were obtained from Wako Pure Chemical Industries (Tokyo).

A Hitachi HPLC system (Tokyo), comprising an L5000LC controller, 655A pump, 655A UV detector and D-2500 integrator, was used. The peptides were eluted on an Inertsil ODS-2 column $(150 \times 4.6 \mathrm{~mm}$ i.d., GL Sciences, Tokyo) with a mixture of acetonitrile $/ 50 \mathrm{mM}$ phosphate buffer ( $\mathrm{pH}$ 6.8) at a constant flow-rate. The column was encased in a Hitachi column oven or in a cooled water bath. A $20 \mu$ l solution of dipeptides ( 30 to $100 \mu \mathrm{g} / \mathrm{ml}$ in water) was injected into the column.

The peak area corresponding to non-isomerized or isomerized molecules is defined as unreacted molecule zone or reacted molecule zone, respectively. Peak area, peak height and retention time were measured by the integrator, but the unreacted molecule zone area shown in Fig. 2 was measured by cutting the profile copy along the zone border and weighing the paper.

\section{Results and Discussion}

Profiles of Leu-Pro and Pro-Leu eluted at lower temperatures

Figure 2 shows the profiles of Leu-Pro and Pro-Leu eluted at a column temperature below $30^{\circ} \mathrm{C}$ at an eluent flow-rate of 1.0 or $0.5 \mathrm{ml} / \mathrm{min}$. A non-convertible peptide of Pro-Leu always produced one symmetrical peak with a constant height at any temperature and flow-rate, while a convertible peptide Leu-Pro produced various profiles. With elution at $10^{\circ} \mathrm{C}$, Leu-Pro produced two sharp peaks of the two isomers, indicating that essentially no isomerization occurred in the column. At a temperature between $16^{\circ}$ and $30^{\circ} \mathrm{C}$, the reaction velocity should be higher, a certain number of the injected molecules should undergo isomerization, thus a reacted molecule zone appeared between the two peaks of unreacted molecule zones. The reacted molecule zone increased with elevation of temperature, adopting the shape of a flat plateau or broad peak, while the unreacted molecule zone decreased in a complementary manner.

The effect of flow-rate on the profile is similar to that of column temperature (Fig. 2). With elution at a slower flow-rate, the retention time should increase, and the probability that a molecule undergoes isomerization should increase; thus the reacted molecule zone increased while the unreacted molecule zone decreased. With elution at $30^{\circ} \mathrm{C}$ at a slow flow-rate of $0.5 \mathrm{ml} / \mathrm{min}$, the unreacted molecule zone disappeared while only a broad peak appeared as a reacted molecule zone, because essentially all the injected molecules underwent isomerization once or more in the column.

\section{Estimation of the reaction velocity in the column}

The unreacted molecule zone of Leu-Pro was fused with the reacted molecule zone but could be separated by bordering them on the assumption that the unreacted

\section{$1.0 \mathrm{ml} / \mathrm{min} \quad 0.5 \mathrm{ml} / \mathrm{min}$}

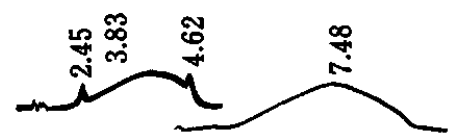

$30^{\circ} \mathrm{C}$

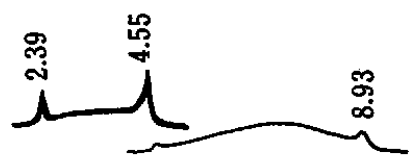

$24^{\circ} \mathrm{C}$



$20^{\circ} \mathrm{C}$
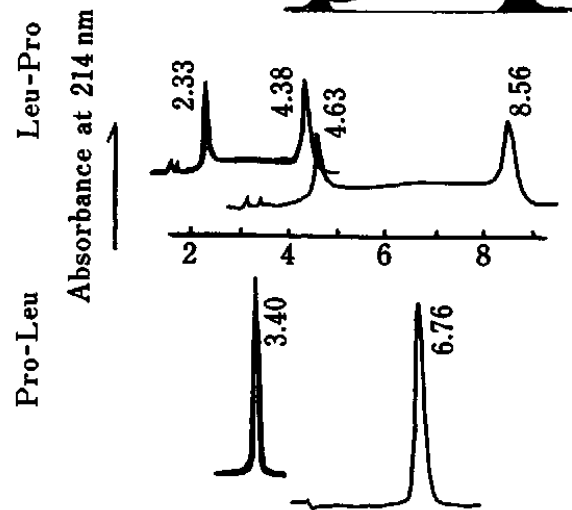

$16^{\circ} \mathrm{C}$

$30^{\circ} \mathrm{C}$

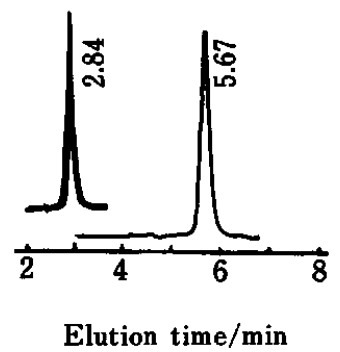

$16^{\circ} \mathrm{C}$

Fig. 2 HPLC profiles of Leu-Pro and Pro-Leu. Column temperature: $16^{\circ}, 20^{\circ}, 24^{\circ}$ or $30^{\circ} \mathrm{C}$. Eluent: acetonitrile/ $50 \mathrm{mM}$ phosphate buffer (pH 6.8) $=10 / 90$. Flow-rate: 1.0 or $0.5 \mathrm{ml} / \mathrm{min}$. Detection: absorbance at $214 \mathrm{~nm}$. The shaded area in one chromatogram (at $20^{\circ} \mathrm{C}$, at $0.5 \mathrm{ml} / \mathrm{min}$ ) shows the unreacted molecule zone (see the text).

molecule zone should be a symmetrical peak as shown in one of the profiles in Fig. 2. Area (\%) of the unreacted molecule zone was determined as described in the Experimental section, and was plotted against the retention time on a semi-logarithmic graph (Fig. 3). The lines were straight, indicating that the reaction followed first-order kinetics. The reaction velocity got higher with elevation of temperature, as expected. Half-lives were estimated from the slopes of these lines, for example, 1.7 and $5.6 \mathrm{~min}$ at $20^{\circ} \mathrm{C}$ for the first and second peak, respectively; then the rate constants $(=0.693 /$ half-life $)$ should be 0.408 and 0.124 per min. The linear plots were extrapolated and coalesced at time zero, indicating that the first and second peak isomers 


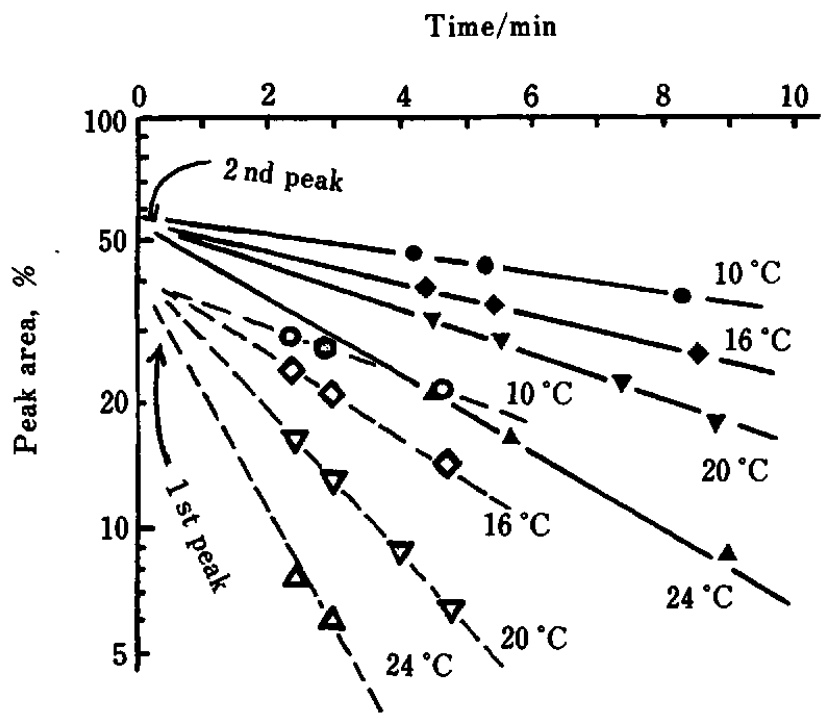

Fig. 3 Relationship between area (\%) and retention time of unreacted Leu-Pro isomer peak. The half-life for the first (---) and second peak (-) was estimated as: 4.7 and $13.5 \mathrm{~min}$ at $10^{\circ} \mathrm{C} ; 3.0$ and $7.8 \mathrm{~min}$ at $16^{\circ} \mathrm{C} ; 1.7$ and $5.6 \mathrm{~min}$ at $20^{\circ} \mathrm{C} ; 1.1$ and $3.2 \mathrm{~min}$ at $24^{\circ} \mathrm{C}$.

were present in an absorbance ratio of $42 \%$ and $56 \%$, respectively, in the sample solution at room temperature before injection onto the column.

\section{Profiles of Leu-Pro and Pro-Leu at higher temperatures}

Leu-Pro produced one broad peak of reacted molecule zone when eluted at a temperature between $40^{\circ}$ and $60^{\circ} \mathrm{C}$ at a 1.0 to $0.3 \mathrm{ml} / \mathrm{min}$ flow-rate. The slower flow-rate gave the higher and sharper peak, which is a common feature of interconvertible isomers and can be explained by the narrower distribution of the greater frequency for randomly occurring phenomenon. Plotting of UV absorbance against elution volume, not against elution time, could demonstrate this effect of flow-rate more clearly (Fig. 4). Pro-Leu, a non-convertible peptide, produced a sharp peak with a constant peak height at any flow-rate tested. In contrast, the peak height $(h)$ of LeuPro was inversely proportional to the square root of flowrate $(v)$ or was proportional to the square root of retention time $\left(t_{\mathrm{r}}\right)$ in the tested range: $h=c \times v^{-1 / 2}=c^{\prime} \times t_{\mathrm{r}}^{1 / 2}$ where $c, c^{\prime}=$ constants (Fig. 5). For example, reduction of flow-rate to a half would produce a $\sqrt{2}(1.41)$-fold higher peak. The theoretical plate number was almost proportionally increasing with the retention time. This linear increase shown in Fig. 5 would be saturating at a further elevated temperature or an extended retention time.

\section{Profiles of Ala-Pro, Val-Pro and Phe-Pro}

Profiles of Ala-Pro, Val-Pro and Phe-Pro are shown in Fig. 6. Effects of temperature and flow-rate on the profiles were basically the same as those of Leu-Pro. The half-life at $20^{\circ} \mathrm{C}$ for the first and second peak isomer



Fig. 4 HPLC profiles of Leu-Pro and Pro-Leu eluted at column temperature of $50^{\circ} \mathrm{C}$ at a $1.0,0.8$ or $0.5 \mathrm{ml} / \mathrm{min}$ flowrate. Absorbance was plotted not against elution time but against elution volume. Other HPLC conditions were the same as those described in Fig. 2. Characteristics of LeuPro peak obtained at $1.0,0.8$ and $0.5 \mathrm{ml} / \mathrm{min}$ flow-rate were: area $238 / 1.0,236 / 0.8,238 / 0.5 \mathrm{mV} \mathrm{s}$; height $6.92,7.70$, $9.60 \mathrm{mV}$; theoretical plate number 295, 372, 570. Those of Pro-Leu peak were: area 261/1.0, 259/0.8, 257/0.5 mV s; height $30.90,31.12,32.00 \mathrm{mV}$; theoretical plate number 7850 , 7530,7950 .

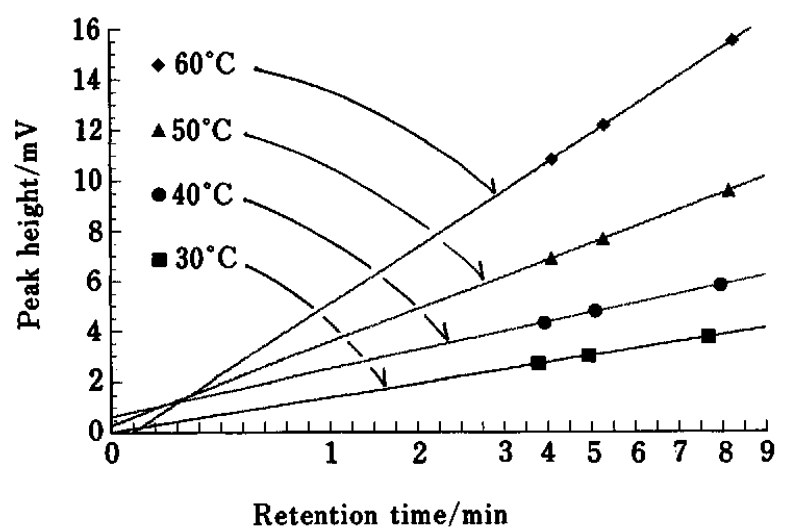

Fig. 5 Relationship between retention time and peak height of Leu-Pro. The horizontal axis shows the square root of retention time. 
(a)
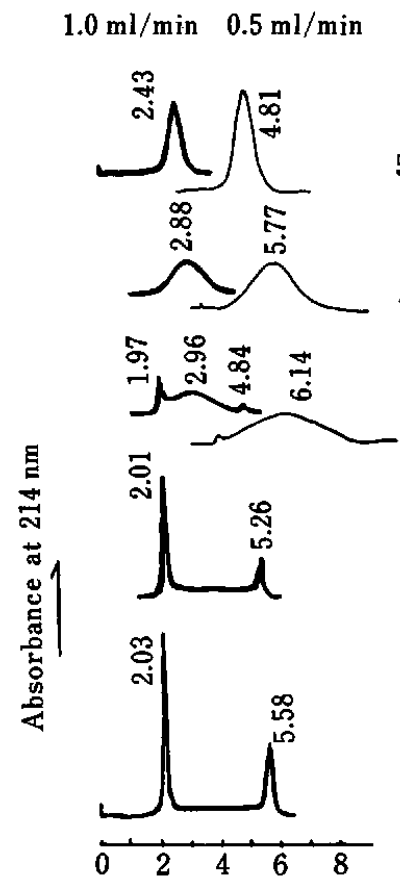

Elution time/min (b)

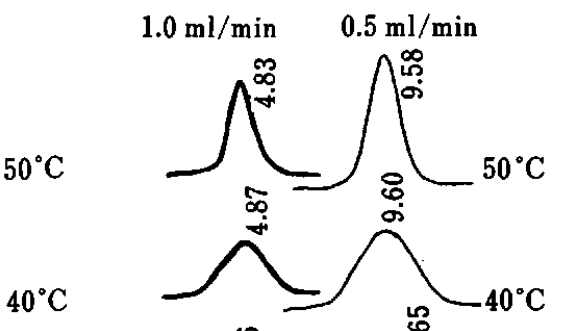

$30^{\circ} \mathrm{C}$

$20^{\circ} \mathrm{C}$



$10^{\circ} \mathrm{C}$
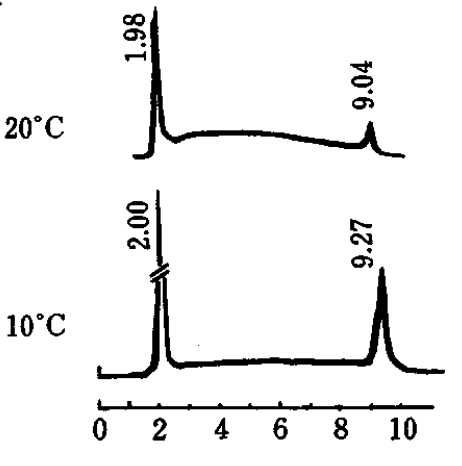

Elution time/min (c)

$1.0 \mathrm{ml} / \mathrm{min} \quad 0.6 \mathrm{ml} / \mathrm{min}$

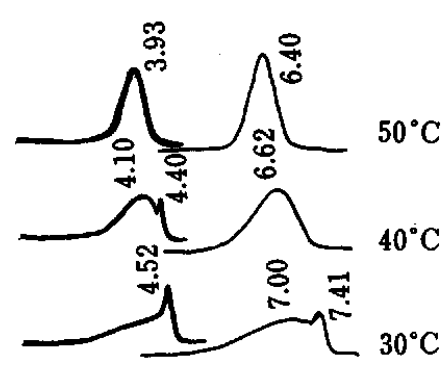

$20^{\circ} \mathrm{C}$

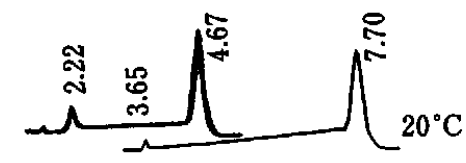

$10^{\circ} \mathrm{C}$



Fig. 6 HPLC profiles of Ala-Pro (a), Val-Pro (b) and Phe-Pro (c). Column temperature: $10^{\circ}, 20^{\circ}, 30^{\circ}, 40^{\circ}$ or $50^{\circ} \mathrm{C}$. Flow-rate: $1.0,0.6$ or $0.5 \mathrm{ml} / \mathrm{min}$. Eluent: acetonitrile $/ 50 \mathrm{mM}$ phosphate buffer $(\mathrm{pH} \mathrm{6.8)}=0 / 100,3.5 / 96.5$ and $15 / 85$ for separation of Ala-Pro, Val-Pro and Phe-Pro, respectively.

was estimated in the manner described before: Ala-Pro 2.0 and $3.0 \mathrm{~min}$; Val-Pro 0.9 and $3.9 \mathrm{~min}$; Phe-Pro 1.2 and $12 \mathrm{~min}$.

\section{Application of the profile analysis methods to the other convertible compounds}

We have presented two methods for the profile analysis: one method shows first-order decrease of nonisomerized molecule number (see Fig. 3), the other shows a square root increase in peak height of isomerized molecules with reduction of flow-rate (see Fig. 5). These two methods for the profile analysis will be easy and practical for distinguishing on-column conversion and for preventing misinterpretation of peculiar profiles.

Both first-order kinetics and the square root increase in peak height were observed in the HPLC profiles of immunosuppressive drugs. ${ }^{2,3}$ In case of the ligandexchange HPLC of sugars, first-order kinetics could be observed; however, the equation showing the square root increase in peak height needs modification because the peak height of non-isomerized sugars was significantly dependent on flow-rate. ${ }^{1}$

\section{References}

1. T. Nishikawa, S. Suzuki, H. Kubo and H. Ohtani, $J$. Chromatogr. A, 720, 167 (1996).

2. T. Nishikawa, H. Hasumi, S. Suzuki, H. Kubo and H. Ohtani, Chromatographia, 38, 359 (1994).

3. T. Nishikawa, H. Hasumi, S. Suzuki, H. Kubo and H. Ohtani, Pharm. Res., 10, 1785 (1993).

4. T. Nishikawa, S. Suzuki and H. Ohtani, J. Antibiotics, 47, 1554 (1994).

5. W. R. Melandar, J. Jacobson and C. Horvath, J. Chromatogr., 234, 269 (1982).

6. J. Jacobson, W. R. Melander, G. Vaisnys and C. Horvath, J. Phys. Chem., 88, 4536 (1984).

7. J. C. Gesquiere, E. Diesis, M. T. Cung and A. Tartar, J. Chromatogr., 478, 121 (1989).

(Received February 23, 1996) (Accepted May 13, 1996) 